\title{
Remote monitoring for better management of LVAD patients: the potential benefits of CardioMEMS
}

\author{
Jesse F. Veenis ${ }^{1}$ (D) Jasper J. Brugts ${ }^{1}$ \\ Received: 15 November 2019 / Accepted: 31 December 2019 / Published online: 24 January 2020 \\ (c) The Author(s) 2020
}

\begin{abstract}
Left ventricular assist devices (LVAD) are frequently used in the treatment of end-stage heart failure (HF), and due to the shortage of heart donors and destination programs, it is likely to keep on growing. Still, LVAD therapy is not without complications and morbidity and rehospitalization rates are high. New ways to improve LVAD care both from the side of the patient and the physician are warranted. Remote monitoring could be a tool to tailor treatment in these patients, as no feedback exists at all about patient functioning on top of the static pump parameters. We aim to provide an overview and evaluation of the novel remote monitoring strategies to optimize LVAD management and elaborate on the opportunities of remote hemodynamic monitoring with CardioMEMS, at home in these patients as the next step to improve care.
\end{abstract}

Keywords Heart failure $\cdot$ LVAD $\cdot$ CardioMEMS $\cdot$ Remote monitoring $\cdot$ Telemonitoring

\section{Introduction}

Epidemiological data on end-stage heart failure (HF) is scarce. Estimations performed by the America Heart Association suggest that $<1 \%$ of all HF patients are in end-stage HF [1]. Other studies estimated that approximately $5-10 \%$ of the HF population develop at some moment in life advanced HF despite optimal medical treatment [2]. These patients become refractory for medical therapy and are frequently hospitalized and have high mortality rates, leaving heart transplantation or left ventricular assist device (LVAD) implantation as the only treatment options. Due to shortness in available heart donors, LVAD implantation rates continue to rise $[3,4]$.

Despite new LVAD designs and technological improvements, LVAD care remains very complex and associated with high mortality and with many rehospitalization and outpatient contacts $[3,5,6]$. The main reason for hospitalization is gastrointestinal (GI) bleeding or LVAD-related (driveline) infection, followed by decompensated HF and arrhythmia [7, 8]. Due to the growing number of patients

Jasper J. Brugts

j.brugts@erasmusmc.nl

1 Department of Cardiology, Thorax Center, Erasmus MC, University Medical Center Rotterdam, Dr. Molewaterplein 40, 3015GD Rotterdam, The Netherlands treated with a LVAD, combined with the high hospitalization and complication rates, LVAD care places a high burden on hospital resources, with many logistical challenges with available hospital beds, as many other departments are not familiar with LVAD devices, so LVAD patients preferably are admitted at a cardiology ward despite non-cardiac admission indications.

It is difficult for pump optimization to be available in a short time at the outpatient clinic and so it is only based on echocardiographic images and static pump parameters.

Patient self-management and remote monitoring is an important part of chronic HF care, to prevent admission. Due to the complexity of LVAD care, remote monitoring has the potential to provide valuable information to help the physician in structured decision making. It has been suggested that remote monitoring of pump parameters, combined with remote monitoring of blood pressure, pacemakerrelated parameters, coagulation values, and driveline exit parameters could improve LVAD care [9]. However, many of these investigations are still unexplored, and not yet tested in large populations. We aim to provide an overview of these new technological advances for the remote monitoring of LVAD patients. 


\section{The LVAD and hemodynamics}

Different LVAD designs, pump mechanisms (axial or centrifugal), and implantation techniques are used. The two most common used LVADs are the HeartMate 3 (HM3, Abbott Inc, Atlanta, GA, USA) and the HVAD (Medtronic Inc, Framingham, MA, USA) (Fig. 1. Both are centrifugal pumps, placed in the pericardial space. The HM3 uses a fully magnetic levitated pump rotor, whereas the HVAD uses passive magnetic and hydrodynamic thrust bearings [10-12].

\section{Common LVAD-related complications}

LVAD care can be lifesaving, however, it is also associated with several LVAD-related complications, such as right ventricular (RV) failure, LVAD-related infection, cardiac arrhythmia, hemolysis and thrombosis, GI bleeding due to angiodysplasia and renal dysfunction [13]. An overview of the incidence rates of common LVAD complications in HM3 and HVAD LVADs is presented in Table 1.

$\mathrm{RV}$ failure is a serious hemodynamic complication, occurring in up to $20-30 \%$ of the LVAD patients [14-17]. Signs of RV failure are elevated central venous pressure (CVP) and manifestations of elevated CVP, such as edema, ascites or increasing renal dysfunction [18].

Tamponade, which can develop shortly after LVAD implantation, is a feared complication, occurring in up to
$20 \%$ of the LVAD patients [19]. Symptoms usually occur in a late stage, and common hemodynamic signs, such as tachycardia, shock or pulsus paradoxus can be masked by the LVAD pump [20]. Late tamponade can be hard to visualize on echocardiography until the patient is in shock, and the first sign might be a drop in pump parameters [21, 22].

GI bleeding is a common complication in LVAD patients, affecting up to $20-30 \%$ of the patients [14-17, 23-25]. GI bleeding has different presenting symptoms, $50 \%$ of patients present with melena, $25 \%$ with unexplained anemia, $15 \%$ with hematochezia and $10 \%$ with hematemesis [26].

LVAD-related infections occur in $10-25 \%$ of the LVAD patients in the first 3 months after LVAD implantation [14-16, 23-25, 27]. Presenting symptoms are fever, erythema at the driveline site, or purulent fluids from the driveline exit site.

Pump thrombosis affects approximately $15 \%$ of axialflow LVAD, and 1\% of centrifugal-flow LVADs [14-16, $23,25]$. Pump thrombosis is characterized by signs of worsening $\mathrm{HF}$ in the patients, which cannot be explained otherwise, abnormal pump parameters and signs of hemolysis in laboratory results, such as elevated LDH [28, 29].
Fig. 1 Schematic presentation of the HeartMate 3 (a) and HeartWare LVAD (c), and close-ups of the pump house and inner work (b, d, resp) Courtesy of Abbott, Inc. and Medtronic, Inc. to provide the illustrations

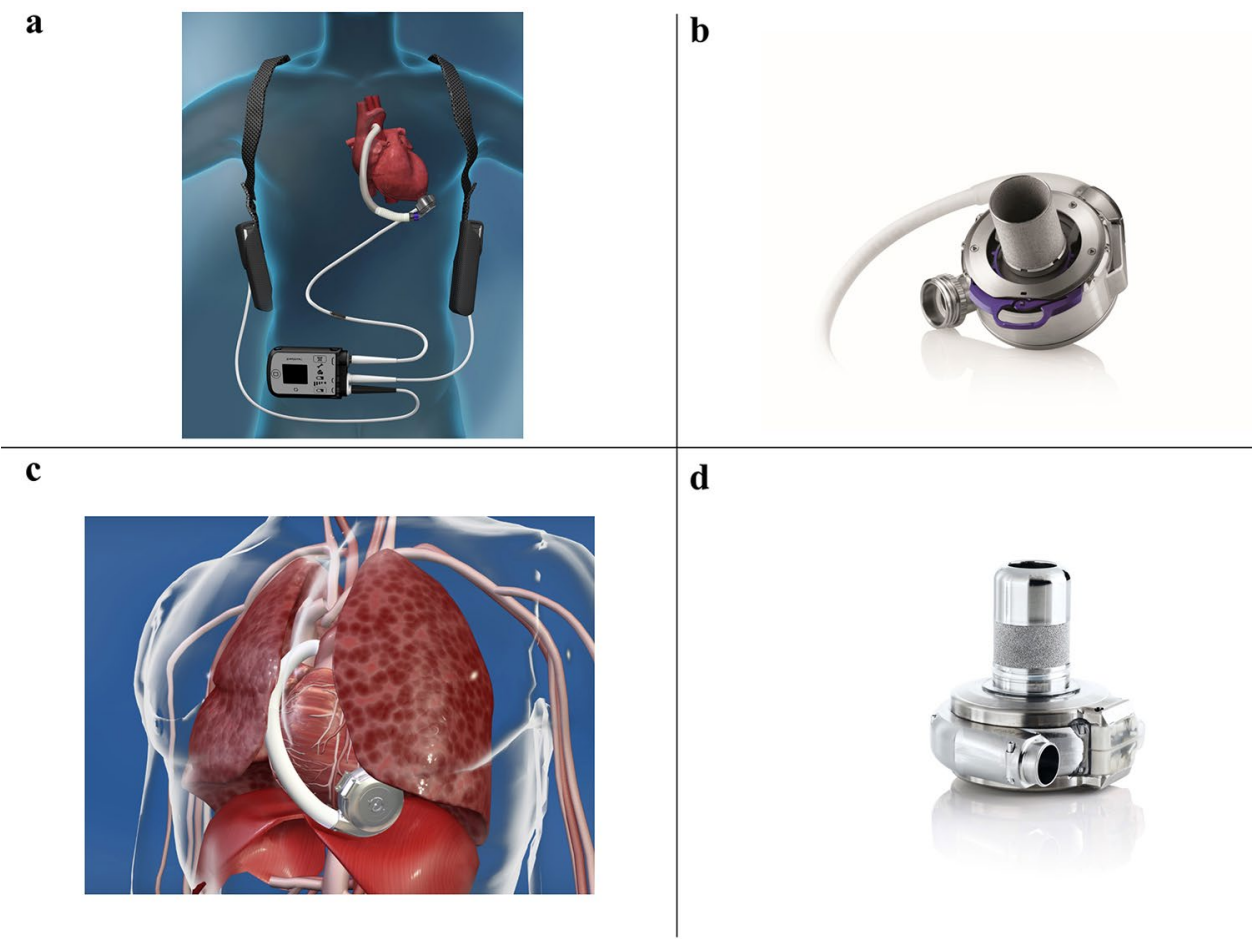


Table 1 Common LVAD-related complications

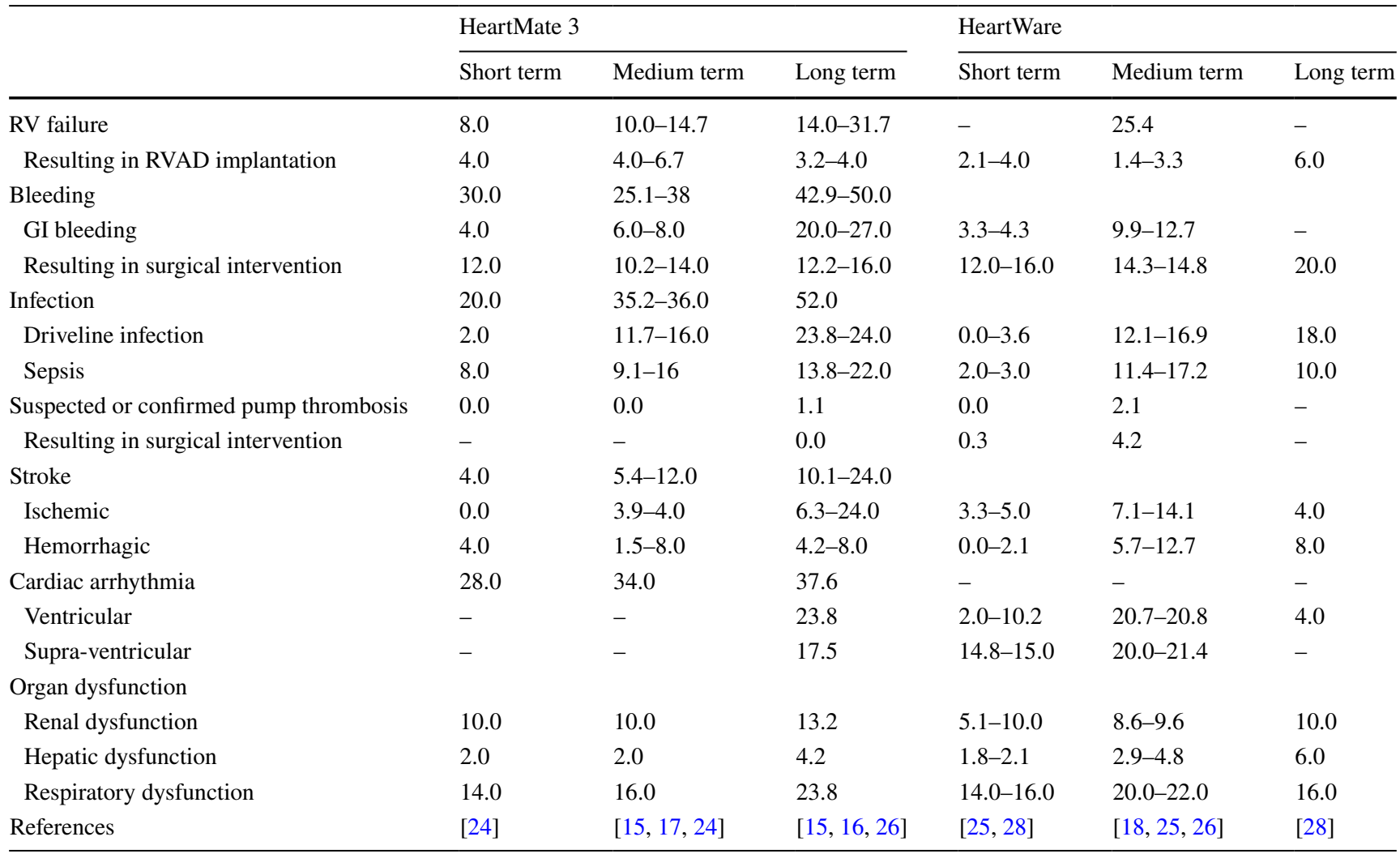

Short term defined as < 1 month; medium term defined as 6-12 months; long term defined as 2 years; - data not available $R V$ right ventricle, $R V A D$ right ventricular assist device, GI Gastrointestinal

\section{Quality of life}

All the above-mentioned LVAD-related complications affect the mortality and morbidity of LVAD patients. However, the complications and the hospitalizations due to these complications also affect the quality of life of LVAD patients. Especially in patients with an LVAD as the destination therapy, quality of life should be the main focus [30]. Remote monitoring of LVAD patients could aid in improving the quality of life of LVAD patients. By earlier detection of LVAD-related complications, earlier intervention is possible, potentially reducing the number of rehospitalizations. Additionally, LVAD settings and the patient's status could be better monitored, allowing for better optimization of the pump settings, improving the pump function [31].

\section{Monitoring strategies}

\section{Non-invasive remote monitoring}

An overview of remote monitoring strategies in LVAD patients is showed in Table 2. One of the keystones in traditional ambulant HF management is the active participation of patients with their medical care team. The use of noninvasive remote monitoring, or structured telephone monitoring in HF patients, has been investigated in multiple trials showing different results [32]. A recent Cochrane review showed that the use of non-invasive and structured telephone remote monitoring reduced mortality and HF-related hospitalizations [32].

At this moment, there is one study that investigated the use of a structured telephone remote monitoring system in LVAD patients [33]. This retrospective study investigated 96 LVAD patients, among who 25 received bi-weekly telephone calls, consisting of an inquiry about LVAD parameters, alarms, blood pressure, INR, body weight, temperature, driveline exit status, symptoms and presence of edema. They found after 2 years a better overall survival in the intervention group ( $89 \%$ vs. $57 \%, p=0.027$ ), however, there was no effect on time free of readmission between the groups.

\section{Remote antithrombotic monitoring}

LVAD care is associated with thromboembolic complications, such as pump thrombosis, which could be a lifethreatening complication, thus showing the need for adequate chronic anticoagulation [7]. However, LVADs are also 
Table 2 Remote monitoring strategies

Number of patients Main findings

Non-invasive remote monitoring

Schloglhöfer et al.

96

Remote antithrombotic monitoring

Dionizovik-Dimanovski et al.

Joshi et al.

41 samples

Bishop et al.

11

Gavalas et al.

956 samples

Remote pump monitoring

Pektok et al.

Kawahito

Bishop et al.

Intrathoracic impedance

Bartoli et al.

Implantable hemodynamic monitoring devices

Feldman et al.

4

Hubert et al.
At 2 years of follow-up, using bi-weekly telephone calls (consisting of an inquiry about LVAD parameters, alarms, blood pressure, INR, body weight, temperature, driveline exit status, symptoms and presence of edema), the overall survival was significantly better compared to standard care ( $89 \%$ vs. $57 \%, p=0.027$ ); but no significant difference in time free of readmission

Moderate correlation between INR measured using a POC device and in a central laboratory(correlation coefficient of 0.83 )

Good correlation between INR measured by a POC device and in a central laboratory (correlation coefficient of 0.96)

Using a POC-INR measurement device at home leads patients to be more often within therapeutic range compared with regular INR measurements at a central laboratory ( $44 \%$ vs. $31 \%, p=0.026$ )

The statistical performance of positive urine hemoglobin to predict $\mathrm{LDH} \geq 600$ IU/L is: sensitivity $60.4 \%$; specificity $85.5 \%$; PPV $42.7 \%$; NPV $92.4 \%$

Demonstrates the feasibility of remote pump parameter monitoring, providing additional information to the treating clinicians

Adding a vibration sensor to an LVAD could adequately detect pieces of silicone, acting like thrombi, at the four most common thrombus locations

In patients with no or minimal AoV regurgitation, adding a specific algorithm could adequately predict AoV opening

Demonstrates the potential utility of intrathoracic impedance measurements in a patient with an LVAD, with an increased intrathoracic impedance preceded intravascular volume depletion and dangerous LVAD dysfunction

Using remote monitored PAP, by the CardioMEMS, leads to a large reduction of PAP and an optimized timing of LVAD implantation compared to those receiving standard care

Significant correlation between left atrial pressure sensor, and pump speed, LV and LA size and pulmonary capillary wedge pressure $(r=0.92-0.99, p<0.05)$

\section{.} . 
echocardiographic analysis and serum lactate dehydrogenase (LDH). LDH is a sign of hemolysis, and probably one of the most reliable markers of pump thrombosis [39, 40]. LDH monitoring is usually only performed during regular outpatient clinic follow-up visits, leading to potential delays in pump thrombosis detection of weeks. Gavalas et al. [41] demonstrated a good correlation between a simple dipstick urine analysis for urine hemoglobin and serum-measured $\mathrm{LDH}$. Absent of urine hemoglobin had a negative predicting value for $\mathrm{LDH} \geq 600 \mathrm{IU} / \mathrm{L}$ (significant hemolysis) of $>90 \%$, thus indicating the potential use for easy remote monitoring at home of pump thrombosis in LVAD patients.

\section{Remote pump monitoring}

Although experience with remote monitoring especially in ICDs and CRTs is growing [42], experience with a remote monitoring function within an LVAD system is limited. However, the first experiences with remote monitoring of pump parameters have been described [43, 44]. The HeartAssist 5 and aVAD LVADs have these remote monitoring functions, allowing to transmit pump parameters, such as pump speed, rounds per minute (rpm) and pump flow, as well as errors, to a website accessible to the care team. This new information can be used in earlier detection of pump complications. Hypovolemia and LVAD thrombosis could be detected by a downward LVAD flow trend [44].

Furthermore, new technologies and algorithms are developed which use the LVAD parameters and help with troubleshooting, and patient monitoring. Detection of vibrations as a sign of mechanical failure is widely used in the biomechanical industry, however, it is not yet used in LVAD management. Kawahito [45] investigated the use of a vibration sensor in combination with an LVAD detect pump thrombosis. This study investigated vibration signals caused by pieces of silicon, acting like actual thrombi, attached at the four most common locations for thrombus in an LVAD: the total area of the bottom of the impeller, an eccentric shape on the bottom of the impeller, a circular shape around the shaft top and an eccentric shape on the top of the impeller. Thrombi at these specific locations can be detected by specific vibration signals, indicating the potential use for early detection of pump thrombosis in LVAD patients.

The aortic valve opening rate is an important aspect of LVAD care. When the aortic valve is not opening the risk of adverse cerebrovascular events increases [46] and commissural fusion can occur, one of the causes of aortic valve regurgitation [47]. Bishop et al. [48] described a novel algorithm to analyze in patients with no or minimal aortic valve regurgitation whether the aortic valve is opening or not. This algorithm uses the electric current waveforms provided by the HeartMate-II LVAD and analyzes this data using a modified Karhunen-Loève transformation. The algorithm could accurately predict aortic valve opening and closing. This algorithm can also be used in an automatic regulation program which can automatically change the rpm settings of the LVAD based on this physiological feedback to maintain a predefined aortic valve opening rate.

\section{Intrathoracic impedance}

Remote intrathoracic impedance monitoring is possible in the newer ICD and CRT devices. A drop in intrathoracic impedance is seen during pulmonary congestion, as an early sign of HF decompensation. Due to the remote monitoring function of newer ICD and CRT devices, the intrathoracic impedance can be used to detect HF decompensation at an earlier stage. Multiple studies investigated whether remote monitoring of intrathoracic impedance could lead to a better outcome in chronic HF patients. A recent systematic review [49] showed that intrathoracic impedance was associated with lower health care costs due to a reduction in planned hospital visits, despite a slight increase in unplanned visits. However, the use of remote impedance monitoring did not affect all-cause or cardiac mortality.

At this moment, there is only one case report [50] describing the use and potential benefits of remote monitoring of intrathoracic impedance in LVAD patients. This patient experienced shortly after LVAD implantation an increase in the impedance as a sign of intravascular fluid depletion. The patient was admitted and treated with fluid repletion and the impedance was increased. This case showed that intrathoracic impedance measurements in LVAD patients might provide some information on their fluid status. However, the use of remote monitoring of impedance in chronic HF holds limited additional value, and it is unclear whether this will be better in LVAD patients.

\section{Implantable hemodynamic monitor devices}

Due to the failure of simple non-invasive and intrathoracic impedance remote monitoring strategies to improve the outcome of chronic HF patients, new, wireless implantable hemodynamic monitor systems were developed. These systems measure filling pressures, and work according to the hypothesis that filling pressures will increase before other signs of decompensated HF occur, as shown in Fig. 2. As has been shown, intracardiac pressures will rise weeks before patients are hospitalized due to decompensated HF [51]. Recently, Abraham provided an overview of multiple implantable hemodynamic monitor devices, which were developed in recent years [52]. In chronic HF patients, one of the most promising techniques is the CardioMEMS system (Abbott Inc, Atlanta, GA, USA) (Fig. 3). This device is implanted in the pulmonary artery during right-heart 
Fig. 2 Hypotheses of pressure monitored and guided heart failure management Reprinted from Abraham [52], 2017, with permission from Elsevier
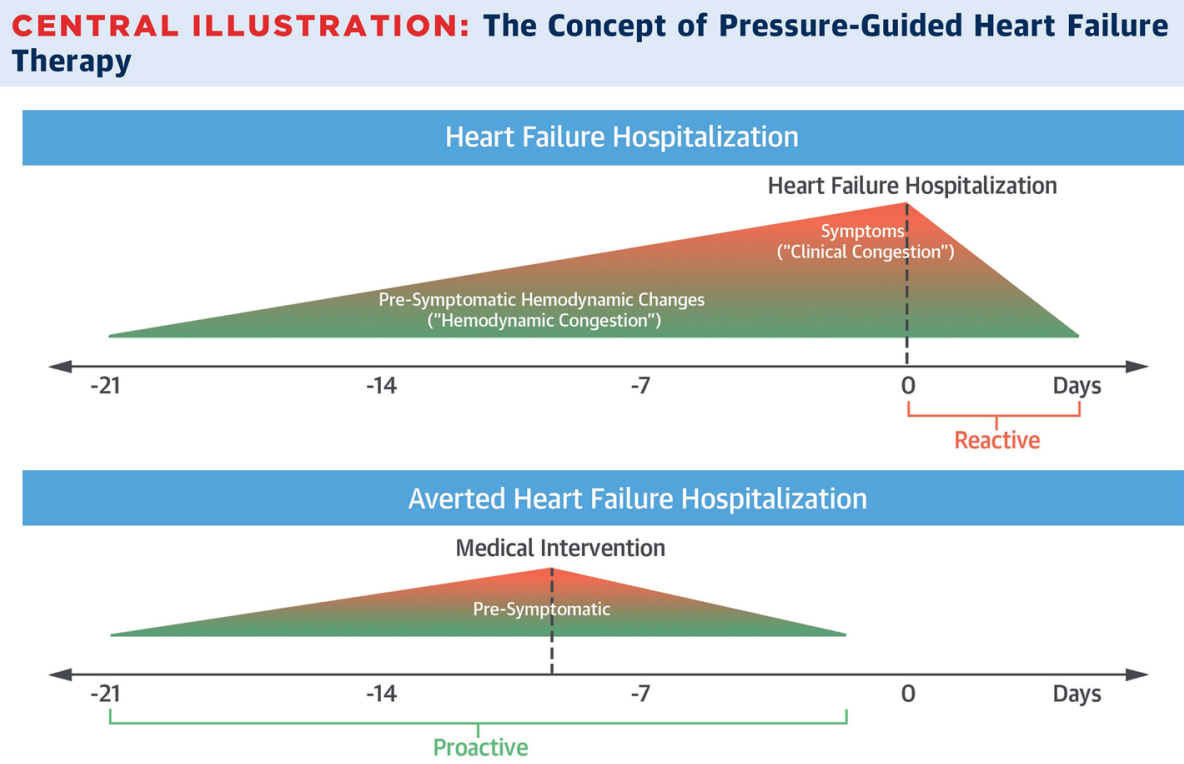

Abraham, W.T. et al. J Am Coll Cardiol. 2017;70(3):389-98.
Fig. 3 CardioMEMS HF system, consisting of the pulmonary artery pressure sensor (a) and the patient electronics system (b) used to take daily pressure readings Courtesy of Abbott, Inc. to provide the illustrations
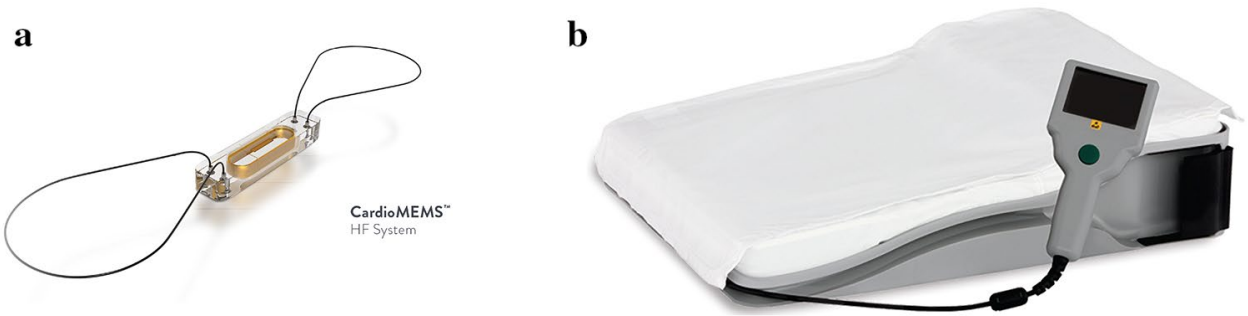

catheterization, and consists of a pressure-sensitive capacitor combined with a coil and can be powered by coupling this electrical circuit with an external antenna. When powered, the capacitor resonates, which is received by the external antenna. When pressure by the pulmonary artery pressure (PAP), is applied, the frequency of resonated energy changes via a characteristic pattern and can be converted into a pressure wave. This system has been shown in clinical trials as well as in real-world clinical practice to be effective in reducing HF hospitalization rates by maintaining normal PAP [53, 54].

A subgroup analysis of the CHAMPION trial, the initial clinical trial investigating the CardioMEMS, consisting of 27 chronic HF patients, who received an LVAD, showed that patients who received an LVAD were sicker, and had a higher PAP when compared to the group who did not receive an LVAD [55]. The intervention group received more medical changes, based on the hemodynamic feedback provided by the CardioMEMS compared to the control group. However, the PAP did not decrease significantly in the patients who received an LVAD, indicating that a lack of decrease of PAP can be a sign of refractory HF, and thus providing useful information in the timing of an LVAD implantation. Post-LVAD implantation, the PAP dropped in both groups, however, using the hemodynamic feedback in the intervention group, the PAP dropped even lower. This indicates that the use of the PAP provided by the CardioMEMS leads to a better and more optimal LVAD management, leading to a better pump function.

Hubbert et al. [56] investigated in four LVAD patients an implantable left atrial pressure (LAP) monitor, the Titan LAP monitoring system (ISS Inc. Ypsilanti, MI). They showed a significant correlation between LAP and pump speed, LV and left atrial size and the pulmonary capillary wedge pressure, thus indicating the potential use of pressures obtained by an implantable hemodynamic monitor for optimization of the pump settings during a ramped speed test. 


\section{Potential impact of implantable hemodynamic monitoring}

We believe that the implantable hemodynamic monitors hold more potential in LVAD patients than currently shown. Using the daily pressure readings, which provide real-time insight into patients' fluid status, the clinicians could optimize patients shortly prior to the LVAD implantation, thereby improving patients' status and their clinical outcome. Also, this hemodynamic information provides direct feedback on medical changes made. We believe that using this hemodynamic feedback will lead to better optimization, thus improving patients' status and potentially improving their clinical outcome [57]. Furthermore, optimizing patients will lead to a better decongestion and thereby better unloading of the RV, thus, reducing the impact of the LVAD implantation on the RV and reducing the risk of RV failure.

Changes in filling pressures post-LVAD implantation might indicate potential postoperative complications. An increase in filling pressures might indicate a tamponade, since the venous return reduces due to inflow obstruction due to elevated pressures in the pericardium. An earlier detection and thereby earlier intervention might prevent latestage tamponade and more severe complications.

Multiple complications, such as pump thrombosis, hemodynamic important arrhythmias or aortic valve regurgitation, will lead to congestion. Similar to chronic HF patients, in LVAD patients filling pressures will rise as a result of congestion [58]. A rise in filling pressures might indicate one of these complications is occurring. Hospitalization and the worsening outcome can potentially be prevented by acting on rising filling pressures. Furthermore, investigating the waveforms and rhythm could provide insight into potential arrhythmias.

A drop in filling pressures might indicate a loss of circulating volume, which might point to a GI bleeding.

LVAD therapy will increase cardiac output, and thereby increase the renal perfusion and resolve the congestion, lowering the renal venous pressure and thereby improving the renal function [59]. Filling pressures might aid in optimizing LVAD therapy, and thus improve the renal function even further.

Fixed pulmonary hypertension is an absolute contraindication for heart transplantation. In these patients, the by ischemic stunned right ventricle will be unable to overcome the elevated afterload and is most likely to fail immediately after heart transplantation. In patients with fixed pulmonary hypertension, LVAD therapy can be used as a bridge to candidacy for heart transplantation, since LVAD therapy is more effective in treating fixed pulmonary hypertension, compared to medical therapy alone [60]. Pulmonary hypertension should be evaluated periodically using a right-heart catheterization, as recommended by the ISHLT guidelines to evaluate whether the patient has become eligible for heart transplantation [61]. However, remote hemodynamic monitoring could replace these periodically right-heart catheterizations, and provide daily feedback on hemodynamic changes. Providing continues insight when a patient could be considered eligible for heart transplantation.

Recently, it has been shown that preforming hemodynamically guided ramp testing could reduce the number of LVAD-related complications and the number of hospitalizations [31, 62, 63]. However, this technique is limited by the need for frequent Swan-Ganz measurements, which increases the risk of bleeding events. Using the hemodynamic information provided by the CardioMEMS, this limitation could be overcome and allowing for easy hemodynamic optimizing of LVAD pump settings.

\section{Future perspectives: design of the HEMO-VAD study to guide LVAD management by hemodynamic feedback}

To investigate the potential impact of an implantable hemodynamic monitor in LVAD patients, we designed the HEMOVAD pilot study[64]. In this study, we will investigate ten consecutive end-stage HF patients, who are accepted for LVAD implantation. These patients will receive prior to LVAD implantation a CardioMEMS device, which will be used for daily hemodynamic monitoring to optimize patients prior to LVAD implantation and monitoring of complications and patient status after LVAD implantation.

\section{Conclusion}

Many remote monitoring strategies are currently investigated and developed for LVAD patients, ranging from non-invasive telephone monitoring programs to implantable hemodynamic monitoring systems. Based on results from trials investigating the use of remote monitoring of regular heart failure, it is warranted to study these devices in LVAD patients. This technique holds the potential to provide additional information for determining the optimal LVAD implantation window, optimizing the patients prior to and post-LVAD implantation, and monitoring for LVADrelated complications to identify the patients most likely to benefit from such therapy and for early discovery of its complications. 


\section{Compliance with ethical standards}

Conflict of interest The author(s) declare that they have no competing interests.

Open Access This article is licensed under a Creative Commons Attribution 4.0 International License, which permits use, sharing, adaptation, distribution and reproduction in any medium or format, as long as you give appropriate credit to the original author(s) and the source, provide a link to the Creative Commons licence, and indicate if changes were made. The images or other third party material in this article are included in the article's Creative Commons licence, unless indicated otherwise in a credit line to the material. If material is not included in the article's Creative Commons licence and your intended use is not permitted by statutory regulation or exceeds the permitted use, you will need to obtain permission directly from the copyright holder. To view a copy of this licence, visit http://creativecommons.org/licenses/by/4.0/.

\section{References}

1. Ammar KA, Jacobsen SJ, Mahoney DW, Kors JA, Redfield MM, Burnett JC Jr, et al. Prevalence and prognostic significance of heart failure stages: application of the American College of Cardiology/American Heart Association heart failure staging criteria in the community. Circulation. 2007;115(12):1563-70.

2. Mancini D, Lietz K. Selection of cardiac transplantation candidates in 2010. Circulation. 2010;122(2):173-83.

3. Briasoulis A, Inampudi C, Akintoye E, Adegbala O, Alvarez $\mathrm{P}$, Bhama J. Trends in utilization, mortality, major complications, and cost after left ventricular assist device implantation in the United States (2009 to 2014). Am J Cardiol. 2018;121(10):1214-8.

4. Patel N, Kalra R, Doshi R, Bajaj NS, Arora G, Arora P. Trends and cost of heart transplantation and left ventricular assist devices: impact of proposed federal cuts. JACC Heart Fail. 2018;6(5):424-32.

5. Kirklin JK, Naftel DC, Pagani FD, Kormos RL, Stevenson LW, Blume ED, et al. Seventh INTERMACS annual report: 15,000 patients and counting. J Heart Lung Transplant. 2015;34(12):1495-504.

6. Kirklin JK, Xie R, Cowger J, de By TMMH, Nakatani T, Schueler $\mathrm{S}$, et al. Second annual report from the ISHLT mechanically assisted circulatory support (IMACS) registry. J Heart Lung Transplant. 2018;37:685-91.

7. Hasin T, Marmor Y, Kremers W, Topilsky Y, Severson CJ, Schirger JA, et al. Readmissions after implantation of axial flow left ventricular assist device. J Am Coll Cardiol. 2013;61(2):153-63.

8. Mehra MR, Salerno C, Cleveland JC, Pinney S, Yuzefpolskaya M, Milano CA, et al. Health care resource use and cost implications in the MOMENTUM 3 long-term outcome study: a randomized controlled trial of a magnetically levitated cardiac pump in advanced heart failure. Circulation. 2018;138:1923-34.

9. Reiss N, Schmidt T, Boeckelmann M, Schulte-Eistrup S, Hoffmann JD, Feldmann C, et al. Telemonitoring of left-ventricular assist device patients-current status and future challenges. J Thorac Dis. 2018;10(Suppl 15):S1794-801.

10. Bourque K, Cotter C, Dague C, Harjes D, Dur O, Duhamel J, et al. Design rationale and preclinical evaluation of the HeartMate 3 left ventricular assist system for hemocompatibility. ASAIO J. 2016;62(4):375-83.
11. Larose JA, Tamez D, Ashenuga M, Reyes C. Design concepts and principle of operation of the HeartWare ventricular assist system. ASAIO J. 2010;56(4):285-9.

12. Burkhoff D, Sayer G, Doshi D, Uriel N. Hemodynamics of mechanical circulatory support. J Am Coll Cardiol. 2015;66(23):2663-74.

13. Kirklin JK, Pagani FD, Kormos RL, Stevenson LW, Blume ED, Myers SL, et al. Eighth annual INTERMACS report: special focus on framing the impact of adverse events. J Heart Lung Transplant. 2017;36(10):1080-6.

14. Schmitto JD, Pya Y, Zimpfer D, Krabatsch T, Garbade J, Rao $\mathrm{V}$, et al. Long-term evaluation of a fully magnetically levitated circulatory support device for advanced heart failure-two-year results from the HeartMate 3 CE Mark Study. Eur J Heart Fail. 2018;21:90-7.

15. Mehra MR, Goldstein DJ, Uriel N, Cleveland JC Jr, Yuzefpolskaya M, Salerno C, et al. Two-year outcomes with a magnetically levitated cardiac pump in heart failure. N Engl J Med. 2018;378(15):1386-95.

16. Gustafsson F, Shaw S, Lavee J, Saeed D, Pya Y, Krabatsch T, et al. Six-month outcomes after treatment of advanced heart failure with a full magnetically levitated continuous flow left ventricular assist device: report from the ELEVATE registry. Eur Heart J. 2018;39(37):3454-60.

17. Bashir J, Legare JF, Freed DH, Cheung A, Rao V, Toma M. Multicentre Canadian experience with the HeartWare ventricular assist device: concerns about adverse neurological outcomes. Can J Cardiol. 2014;30(12): 1662-7.

18. Lampert BC, Teuteberg JJ. Right ventricular failure after left ventricular assist devices. J Heart Lung Transplant. 2015;34(9):1123-30.

19. Kohmoto T, Oz MC, Naka Y. Late bleeding from right internal mammary artery after HeartMate left ventricular assist device implantation. Ann Thorac Surg. 2004;78(2):689-91.

20. Topilsky Y, Price TN, Atchison FW, Joyce LD. Atypical tamponade hemodynamic in a patient with temporary left ventricular assist device. Interact Cardiovasc Thorac Surg. 2011;12(5):832-4.

21. Jett GK. ABIOMED BVS 5000: experience and potential advantages. Ann Thorac Surg. 1996;61(1):301-4 (discussion 11-3).

22. Smart K, Jett GK. Late tamponade with mechanical circulatory support. Ann Thorac Surg. 1998;66(6):2027-8.

23. Netuka I, Sood P, Pya Y, Zimpfer D, Krabatsch T, Garbade J, et al. Fully magnetically levitated left ventricular assist system for treating advanced HF: a multicenter study. J Am Coll Cardiol. 2015;66(23):2579-89.

24. Slaughter MS, Pagani FD, McGee EC, Birks EJ, Cotts WG, Gregoric I, et al. HeartWare ventricular assist system for bridge to transplant: combined results of the bridge to transplant and continued access protocol trial. J Heart Lung Transplant. 2013;32(7):675-83.

25. Aaronson KD, Slaughter MS, Miller LW, McGee EC, Cotts WG, Acker MA, et al. Use of an intrapericardial, continuous-flow, centrifugal pump in patients awaiting heart transplantation. Circulation. 2012;125(25):3191-200.

26. Marsano J, Desai J, Chang S, Chau M, Pochapin M, Gurvits GE. Characteristics of gastrointestinal bleeding after placement of continuous-flow left ventricular assist device: a case series. Dig Dis Sci. 2015;60(6):1859-67.

27. Strueber M, O'Driscoll G, Jansz P, Khaghani A, Levy WC, Wieselthaler GM, et al. Multicenter evaluation of an intrapericardial left ventricular assist system. J Am Coll Cardiol. 2011;57(12):1375-82.

28. Shah P, Tantry US, Bliden KP, Gurbel PA. Bleeding and thrombosis associated with ventricular assist device therapy. J Heart Lung Transplant. 2017;36(11):1164-73. 
29. Stulak JM, Sharma S, Maltais S. Management of pump thrombosis in patients with left ventricular assist devices. Am J Cardiovasc Drugs. 2015;15(2):89-94.

30. Adams EE, Wrightson ML. Quality of life with an LVAD: a misunderstood concept. Heart Lung. 2018;47(3):177-83.

31. Uriel N, Burkhoff D, Rich JD, Drakos SG, Teuteberg JJ, Imamura $\mathrm{T}$, et al. Impact of hemodynamic ramp test-guided HVAD speed and medication adjustments on clinical outcomes. Circ Heart Fail. 2019;12(4):e006067.

32. Inglis SC, Clark RA, Dierckx R, Prieto-Merino D, Cleland JG. Structured telephone support or non-invasive telemonitoring for patients with heart failure. Cochrane Database Syst Rev. 2015;10:CD007228.

33. Schloglhofer T, Horvat J, Moscato F, Hartner Z, Necid G, Schwingenschlogl $\mathrm{H}$, et al. A standardized telephone intervention algorithm improves the survival of ventricular assist device outpatients. Artif Organs. 2018;42:961-9.

34. Demirozu ZT, Radovancevic R, Hochman LF, Gregoric ID, Letsou GV, Kar B, et al. Arteriovenous malformation and gastrointestinal bleeding in patients with the HeartMate II left ventricular assist device. J Heart Lung Transplant. 2011;30(8):849-53.

35. Heneghan C, Ward A, Perera R, Self-Monitoring Trialist C, Bankhead C, Fuller A, et al. Self-monitoring of oral anticoagulation: systematic review and meta-analysis of individual patient data. Lancet. 2012;379(9813):322-34.

36. Dionizovik-Dimanovski M, Levin AP, Fried J, Mody KP, Simonich E, Garan AR, et al. Correlation between home INR and core laboratory INR in patients supported with continuous-flow left ventricular assist devices. ASAIO J. 2015;61(4):386-90.

37. Joshi A, Smith D, Arora M, Poston R. Anticoagulant monitoring in ventricular assist device patients: a feasibility study. Interact Cardiovasc Thorac Surg. 2008;7(6):1035-8.

38. Bishop MA, Streiff MB, Ensor CR, Tedford RJ, Russell SD, Ross PA. Pharmacist-managed international normalized ratio patient self-testing is associated with increased time in therapeutic range in patients with left ventricular assist devices at an academic medical center. ASAIO J. 2014;60(2):193-8.

39. Uriel N, Morrison KA, Garan AR, Kato TS, Yuzefpolskaya M, Latif F, et al. Development of a novel echocardiography ramp test for speed optimization and diagnosis of device thrombosis in continuous-flow left ventricular assist devices: the Columbia ramp study. J Am Coll Cardiol. 2012;60(18):1764-75.

40. Shah P, Mehta VM, Cowger JA, Aaronson KD, Pagani FD. Diagnosis of hemolysis and device thrombosis with lactate dehydrogenase during left ventricular assist device support. J Heart Lung Transplant. 2014;33(1):102-4.

41. Gavalas MV, Breskin A, Yuzefpolskaya M, Eisenberger A, Castagna F, Demmer RT, et al. Discriminatory performance of positive urine hemoglobin for detection of significant hemolysis in patients with continuous-flow left ventricular assist devices. J Heart Lung Transplant. 2017;36(1):59-63.

42. Varma N, Ricci RP. Telemedicine and cardiac implants: what is the benefit? Eur Heart J. 2013;34(25):1885-95.

43. Pektok E, Demirozu ZT, Arat N, Yildiz O, Oklu E, Eker D, et al. Remote monitoring of left ventricular assist device parameters after HeartAssist-5 implantation. Artif Organs. 2013;37(9):820-5.

44. Hohmann S, Veltmann C, Duncker D, Konig T, Berliner D, Hanke $\mathrm{J}$, et al. Initial experience with telemonitoring in left ventricular assist device patients. J Thorac Dis. 2019;11(Suppl 6):S853-63.

45. Kawahito K. Transformation of vibration signals in rotary blood pumps: the diagnostic potential of pump failure. J Artif Organs. 2013;16(3):393-6.

46. Lazar RM, Shapiro PA, Jaski BE, Parides MK, Bourge RC, Watson JT, et al. Neurological events during long-term mechanical circulatory support for heart failure: the Randomized Evaluation of Mechanical Assistance for the Treatment of
Congestive Heart Failure (REMATCH) experience. Circulation. 2004;109(20):2423-7.

47. Saito T, Wassilew K, Gorodetski B, Stein J, Falk V, Krabatsch T, et al. Aortic valve pathology in patients supported by continuousflow left ventricular assist device. Circ J. 2016;80(6):1371-7.

48. Bishop CJ, Mason NO, Kfoury AG, Lux R, Stoker S, Horton K, et al. A novel non-invasive method to assess aortic valve opening in HeartMate II left ventricular assist device patients using a modified Karhunen-Loeve transformation. J Heart Lung Transplant. 2010;29(1):27-31.

49. Klersy C, Boriani G, De Silvestri A, Mairesse GH, Braunschweig F, Scotti V, et al. Effect of telemonitoring of cardiac implantable electronic devices on healthcare utilization: a meta-analysis of randomized controlled trials in patients with heart failure. Eur J Heart Fail. 2016;18(2):195-204.

50. Bartoli CR, Vessels KM, McCants KC. Increased intrathoracic impedance may predict adverse events in LVAD patients. J Card Surg. 2013;28(5):616-8.

51. Zile MR, Bennett TD, St John Sutton M, Cho YK, Adamson PB, Aaron MF, et al. Transition from chronic compensated to acute decompensated heart failure: pathophysiological insights obtained from continuous monitoring of intracardiac pressures. Circulation. 2008;118(14):1433-41.

52. Abraham WT, Perl L. Implantable hemodynamic monitoring for heart failure patients. J Am Coll Cardiol. 2017;70(3):389-98.

53. Abraham WT, Stevenson LW, Bourge RC, Lindenfeld JA, Bauman JG, Adamson PB, et al. Sustained efficacy of pulmonary artery pressure to guide adjustment of chronic heart failure therapy: complete follow-up results from the CHAMPION randomised trial. Lancet. 2016;387(10017):453-61.

54. Heywood JT, Jermyn R, Shavelle D, Abraham WT, Bhimaraj A, Bhatt K, et al. Impact of practice-based management of pulmonary artery pressures in 2000 patients implanted with the CardioMEMS sensor. Circulation. 2017;135(16):1509-17.

55. Feldman DS, Moazami N, Adamson PB, Vierecke J, Raval N, Shreenivas S, et al. The utility of a wireless implantable hemodynamic monitoring system in patients requiring mechanical circulatory support. ASAIO J. 2018;64(3):301-8.

56. Hubbert L, Baranowski J, Delshad B, Ahn H. Left atrial pressure monitoring with an implantable wireless pressure sensor after implantation of a left ventricular assist device. ASAIO J. 2017;63(5):e60-5.

57. Cowger J, Shah P, Stulak J, Maltais S, Aaronson KD, Kirklin JK, et al. INTERMACS profiles and modifiers: heterogeneity of patient classification and the impact of modifiers on predicting patient outcome. J Heart Lung Transplant. 2016;35(4):440-8.

58. Abraham WT, Adamson PB, Bourge RC, Aaron MF, Costanzo MR, Stevenson LW, et al. Wireless pulmonary artery haemodynamic monitoring in chronic heart failure: a randomised controlled trial. Lancet. 2011;377(9766):658-66.

59. Verma S, Bassily E, Leighton S, Mhaskar R, Sunjic I, Martin A, et al. Renal function and outcomes with use of left ventricular assist device implantation and inotropes in end-stage heart failure: a retrospective single center study. J Clin Med Res. 2017;9(7):596-604.

60. Kumarasinghe G, Jain P, Jabbour A, Lai J, Keogh AM, Kotlyar $\mathrm{E}$, et al. Comparison of continuous-flow ventricular assist device therapy with intensive medical therapy in fixed pulmonary hypertension secondary to advanced left heart failure. ESC Heart Fail. 2018;5(4):695-702.

61. Mehra MR, Canter CE, Hannan MM, Semigran MJ, Uber PA, Baran DA, et al. The 2016 International Society for Heart Lung Transplantation listing criteria for heart transplantation: a 10-year update. J Heart Lung Transplant. 2016;35(1):1-23.

62. Imamura T, Nguyen A, Kim G, Raikhelkar J, Sarswat N, Kalantari S, et al. Optimal haemodynamics during left ventricular assist device support are associated with reduced 
haemocompatibility-related adverse events. Eur J Heart Fail. 2019;21(5):655-62.

63. Imamura T, Jeevanandam V, Kim G, Raikhelkar J, Sarswat N, Kalantari S, et al. Optimal hemodynamics during left ventricular assist device support are associated with reduced readmission rates. Circ Heart Fail. 2019;12(2):e005094.

64. Veenis JF, Manintveld OC, Constantinescu AA, Caliskan K, Birim $\mathrm{O}$, Bekkers JA, et al. Design and rationale of haemodynamic guidance with CardioMEMS in patients with a left ventricular assist device: the HEMO-VAD pilot study. ESC Heart Fail. 2019;6:194-201.

Publisher's Note Springer Nature remains neutral with regard to jurisdictional claims in published maps and institutional affiliations. 